\title{
Matemáticas en el bachillerato internacional y bachillerato general unificado de Ecuador. Un estudio crítico
}

\author{
Mathematics in the international baccalaureate and unified general \\ baccalaureate of Ecuador. A critical study
}

\author{
Luis Marcelo Mantilla-Falcón. ${ }^{1}$, Diego Israel Romero-Castro. ${ }^{2}$, Eugenia Antonieta \\ Fonseca-Gómez. ${ }^{3}$ \& Mery Susana Mantilla-Falcón. ${ }^{4}$
}

\begin{abstract}
.
DOI: https://doi.org/10.33262/cienciadigital.v5i4.1867

Education is a social process that is constantly evolving. National education systems are constantly adjusting their curricular designs to adapt to the present reality in accordance with the new demands of the economy, science and technology. Objective: this study analyzes and discusses the divergences and convergences between the Unified General Baccalaureate of Ecuador and the International Baccalaureate, which has been present in the country since 2010. Methodology: It is a qualitative research of interpretative nature with a deep content analysis from the perspective of hermeneutics, to systematize the information and base the criteria and discussion. The researchers chose the mathematics curriculum because of their teaching experience and because it is one of the subjects that is evaluated internationally through the PISA tests. Contributions: Once the analysis and

\footnotetext{
${ }^{1}$ Doctor en Ciencias de la Educación, Magister en Docencia Universitaria y Administración Educativa, Magister en Ciencias de la Educación, Maestrante en Pedagogía; Docente en la Universidad Técnica de Ambato, Ambato-Ecuador, luismmantilla@uta.edu.ec, https://orcid.org/0000-0002-8209-7365

${ }^{2}$ Ingeniero en Electrónica e Instrumentación; Docente en la Unidad Educativa Aloasí, Latacunga-Ecuador diegoi.romero@educacion.gob.ec, https://orcid.org/0000-0003-4982-2809

${ }^{3}$ Licenciada en Docencia y Gestión de Educación Básica, Docente en la Unidad Educativa San Francisco de Asis, Latacunga-Ecuador, eafonsecag @ pucesa.edu.ec, https://orcid.org/0000-0001-9926-4556

4 Ingeniera Comercial, Docente en el Instituto Tecnológico Edupraxis, Ambato-Ecuador, msmantilla2611@ @otmail.com, https://orcid.org/0000-0002-2474-0076
} 
interpretation were done, the results show profound differences between the two baccalaureates in terms of content distribution, time, mastery of skills with performance criteria, skills and evaluation processes. Conclusion: It is concluded that the International Baccalaureate presents better learning results, due to its organization, level of rigor and dynamic methodology, which enables students to continue their university career in any institution of higher education in the country and the world.

Keywords: skill, teaching, educational strategies, assessment, mathematics.

\section{Resumo.}

A educação é um processo social em permanente evolução. Os sistemas educacionais nacionais ajustam constantemente seus desenhos curriculares para se adaptarem à realidade atual de acordo com as novas demandas da economia, da ciência e da tecnologia. Objetivo: Este estudo analisa e discute as divergências e convergências entre o Bacharelado Geral Unificado do Equador e o Bacharelado Internacional que está presente no país desde 2010. Metodologia: É uma pesquisa qualitativa de natureza interpretativa com uma profunda análise de conteúdo do ponto de vista hermenêutico, para sistematizar as informações e fundamentar os critérios e discussão. Os pesquisadores escolheram o currículo de matemática por causa de sua experiência de ensino e por ser uma das disciplinas avaliadas internacionalmente por meio dos testes do PISA. Contribuições: Uma vez feita a análise e interpretação, os resultados mostram profundas diferenças entre as duas escolas de ensino médio em termos de distribuição de conteúdo, tempo, domínio de habilidades com critérios de desempenho, habilidades e processos de avaliação. Conclusão: Conclui-se que o Bacharelado Internacional apresenta melhores resultados de aprendizagem, devido à sua organização, nível de rigor e metodologia dinâmica, que permite ao aluno prosseguir a sua carreira universitária em qualquer instituição de ensino superior do país e do mundo.

Palavras-chave: habilidade, ensino, estratégias educacionais, avaliação, matemática

\section{Resumen.}

La educación es un proceso social que está en permanente evolución. Los sistemas educativos nacionales ajustan constantemente sus diseños curriculares para acoplarse a la realidad presente en concordancia a las nuevas exigencias de la economía, la ciencia y la tecnología. Objetivo: El presente estudio analiza y discute las divergencias y convergencias entre el bachillerato general unificado del Ecuador y el bachillerato internacional que tiene su presencia en el país desde el año 2010. Metodología: Se trata de una investigación cualitativa de carácter interpretativo con un profundo análisis de contenido desde la óptica de la hermenéutica, para sistematizar la información y fundamentar los criterios y discusión. Los investigadores escogieron el currículo de las matemáticas por su experiencia docente y porque es una de las asignaturas que se evalúa a nivel internacional a través de las pruebas PISA. Aportes: Una vez hecho el análisis y la interpretación, los resultados evidencian profundas diferencias entre los dos bachilleratos tanto en distribución de contenidos, tiempos, dominio de destrezas con criterios de desempeño, habilidades y procesos de evaluación. Conclusión: Se concluye 
que el bachillerato internacional presenta mejores resultados de aprendizaje, por su organización, nivel de rigurosidad y metodología dinámica, este le habilita al estudiante para continuar con su carrera universitaria en cualquier institución de educación superior del país y del mundo.

Palabras claves: destreza, docencia, estrategias educativas, evaluación, matemáticas.

\section{Introducción.}

La educación ecuatoriana ha tenido cambios importantes en sus currículos a lo largo de la historia en todas las asignaturas, por tanto, las matemáticas no han estado fuera de esta realidad. Desde esta perspectiva se busca contrastar el currículo en Matemática del bachillerato ecuatoriano a partir de la última reforma (2016) frente a la vigencia del currículo en Matemática del Bachillerato Internacional presente en el Ecuador desde el 2011.

La investigación recurre a un amplio análisis interpretativo (hermenéutica) en función del estudio pormenorizado y muy riguroso de los dos diseños curriculares en Matemáticas para contrastar las fortalezas, debilidades, oportunidades, semejanzas y diferencias que cada uno presenta en su estructura, forma, secuencia, metodología, evolución, cumplimiento de destrezas con criterio de desempeño y habilidades.

Las pruebas PISA evidencian que los estudiantes de Bachillerato Internacional, BI, alcanzan mejores resultados, en consecuencia, ¿Qué ventajas y beneficios ofrece en BI, que no pueda hacerlo el Bachillerato General Unificado, BGU, en la asignatura de Matemática? Los resultados y juicios de valor, producto del análisis, servirán para que las autoridades respectivas reorienten la política educativa en cuestión de planificación curricular y logren insertar al Ecuador en los estándares de calidad internacionales.

\section{Marco teórico}

En las últimas tres décadas el Ecuador ha tenido algunas reformas curriculares de su sistema educativo, particularmente la de 1996, 2010 y 2016, siempre con la finalidad de mejorar la calidad de la educación y converger hacia la integralidad entre sistemas y componentes del hecho educativo (Herrera Pavo \& Cochancela Patiño, 2020), en efecto las reformas realizan ajustes y modificaciones orientadas a acoplarse a las presiones actuales que el mundo moderno exige, tanto en adelanto científico, pedagógico y tecnológico.

No basta solo con un listado de contenidos que evalúen cuánto sabe el estudiante, los currículos han orientado su horizonte de actuación tomando en cuenta lo procedimental, lo actitudinal, lo valorativo y transformacional, en una suerte de bucle, que convierta el accionar educativo en un sistema totalmente interrelacionado considerando al estudiante como el centro de atención desde su integralidad bio-psico-social e intelectual. 
Para entender la dinámica del currículo en las matemáticas, primero es fundamental comprender la idea básica y general de lo que es un currículo que en palabras de Perlo Pachega (2019) "se trata de un proyecto pedagógico amplio y complejo, que involucra una concepción del ser, de la realidad y la naturaleza del encuentro humano" (p. 70), en la misma línea de criterio el currículo tiene que responder a intencionalidades de la institución de cara a las transformaciones sociales, culturales, políticas y económicas (Malagón Plata, Rodríguez Rodríguez, \& Ñáñez Rodríguez, 2019).

En definitiva, el currículo:

...es concebido como dispositivo central para reorientar el quehacer escolar en relación con un modelo o tendencia pedagógica, en cumplimiento de objetivos predeterminados, que limitan su comprensión a un plan de estudios o a un listado de contenidos de lo que se ha de enseñar (Niño Zafra, 2013, pág. 11).

Para avanzar en procesos francos de transformación y desarrollo educativo, el currículo debe ser el elemento central a tomar en cuenta y de esa manera adaptar la nueva escuela a la realidad actual, porque la función principal del currículo es permitir trasladar los principios y los propósitos pedagógicos al aula de clase, esto significa sintetizar la praxis pedagógica y orientar la acción y la reflexión que genera la misma; el currículo pone en diálogo la teoría y la práctica, la reflexión y la acción, los principios pedagógicos más generales y las didácticas de aula (de Zubiría Samper, 2013).

En definitiva, el currículo es un corpus de objetivos de aprendizaje debidamente seleccionados que busca generar experiencias apropiadas que se irán acumulando con el tiempo y deberán ser evaluadas en su momento, deben ser planificadas, dirigidas y ejecutadas por la institución educativa en función del tipo de ser humano a formarse que encaje en la sociedad presente (Ortiz Ocaña, 2014).

Los modelos curriculares sufren cambios constantes y en este proceso evolutivo de las reformas curriculares en el Ecuador no ha sido la excepción, por ejemplo, en el año 1996 se propone la Reforma Curricular Consensuada para la Educación Básica como producto de una reforma fallida previa de 1994, basada en un enfoque eminentemente conceptual. La reforma de 1996 tenía entre otras, las siguientes características: se incorporó a la educación básica, la educación inicial, no tuvo un enfoque pedagógico definido y se introduce las nociones de destrezas y valores en el currículo como un atisbo de integralidad en el proceso de enseñanza aprendizaje (Herrera Pavo \& Cochancela Patiño, 2020).

De acuerdo con los mismos autores, para el 2010, la reforma integra en su estructura una visión antropológica y filosófica desde el Sumak Kawsay o el Buen Vivir, considera una serie de orientaciones metodológicas, el establecimiento de indicadores de evaluación y una amplia formulación de estándares de aprendizaje. El aparecimiento de ejes transversales fue muy significativo, entre ellos: interculturalidad, formación de una ciudadanía democrática, protección del medio ambiente, cuidado de la salud y de los 
hábitos de recreación de los estudiantes, y; educación sexual de los jóvenes (Ministerio de Educación, 2010).

En la última reforma curricular del año 2016 la característica central es la fijación del perfil de salida del bachiller, producto de un trabajo colaborativo entre la comunidad educativa y el sector productivo. Se reestructuró los subniveles como sigue: Preparatoria 1 año; Elemental, 3 años; Media, 3 años y Superior, 3 años, de educación General Básica y sumado al nivel de Bachillerato General Unificado BGU 3 años más (Ministerio de Educación, 2016a). Otro hecho importante es la secuenciación de contenidos por áreas y bloques en función de los subniveles educativos de desarrollo, "Esta estructura permite que los contenidos puedan moverse dentro del bloque, a través de los diferentes niveles y subniveles educativos, en función de las necesidades de cada contexto educativo específico" (Herrera Pavo \& Cochancela Patiño, 2020, pág. 6). Se mantiene el concepto u orientación de destrezas con criterios de desempeño y se profundiza en la triangulación de los aspectos conceptual, cognitivo, procedimental y actitudinal.

En lo concerniente al Bachillerato Internacional, BI, su vinculación al Ecuador tienen algunas consideraciones, por ejemplo, el 9 de febrero del 2006 se firma el Memorando de Acuerdos Mutuos y Entendimiento con la Organización de Bachillerato Internacional (IBO, por sus siglas en inglés) de Ginebra, Suiza, para la inserción del BI en colegios fiscales del país. Este programa se caracteriza por su alta exigencia académica, rigurosidad evaluativa que le prepara al estudiante para la continuidad de sus estudios en universidades nacionales e internacionales en cualquier parte del mundo (Instituto Nacional de Evaluación Educativa, INEVAL, 2018a).

$\mathrm{El} \mathrm{BI}$, es considerado como un programa preuniversitario que tiene como parte de sus objetivos de formación construir un estudiante con mentalidad internacional, consciente de su condición humana, comprometido con la naturaleza, que piensa en un mundo mejor con humanidad y responsabilidad por lo que se compromete a ser: indagador, de mente abierta, informado e instruido, solidario, pensador, audaz, buen comunicador, equilibrado, íntegro y reflexivo, es decir, que con estos atributos el estudiante con BI será un protagonista transformador de su realidad local, nacional e internacional (International Baccalaureate Organization, 2014).

El BI cuenta con cuatro programas que en su orden son: Programa de la Educación Primaria (PEP); Programa de los años Intermedios (PAI), Programa del Diploma (PD) y Programa de Orientación Profesional (POP) cada uno de ellos con su estructura curricular, sus políticas y sus fines bien definidos delimitados pero interrelacionados (International Baccalaureate Organization, 2019).

Desde la concepción de la enseñanza se basan en seis pilares fundamentales: la indagación; comprensión conceptual; se desarrolla en contextos locales y globales; se centra en el trabajo en equipo y colaboración eficaz; elimina las barreras para el aprendizaje, y está guiado por la evaluación. Asimismo, desde el enfoque del aprendizaje, el campo de acción de este bachillerato está centrado en las habilidades distribuidas en cinco categorías: de pensamiento, de investigación, de comunicación, sociales y, de 
argumentación, acciones que convierten al estudiante en un ente con actitud activa, crítica y reflexiva capaz de aprender a lo largo de su vida (International Baccalaureate Organization, 2019).

Para comprender la complejidad del currículo y aterrizar en la práctica pedagógica de la matemática en el aula, es importante describir sus características y contexto en términos de interrelación de la planificación a nivel macro, meso y micro curricular y el desarrollo mismo de la asignatura y sus implicaciones en la formación del estudiante, esto significa que, el currículo de las matemáticas, que generalmente ha sido tradicionalista, memorístico debe convertirse en un espacio reflexivo, crítico y analítico que desarrolle procesos mentales superiores para responder los problemas de la realidad actual (Arévalo Duarte \& Gamboa Suárez, 2015), en consecuencia, la matemática debe ser la disciplina científica capaz de movilizar la estructura intelectiva del estudiante que le permita, con esos conocimientos, tomar decisiones acertadas para solucionar toda la gama de escenarios que se presente en la vida contextualizada.

"El docente de hoy requiere comprender la necesidad e importancia de un currículo y una didáctica contextualizada y desarrolladora en función de lograr la excelencia académica, a partir del diseño de los planes y programas integrales, flexibles y contextualizados" (Ortiz Ocaña, 2014, pág. 13), por tanto, enseñar matemáticas no es tarea fácil, su aplicación en la práctica pedagógica requiere de habilidades y competencias más exigentes porque las evidencias determinan que la pérdida de la asignatura es alta, ya sea por carencia de metodologías innovadoras, rigidez en el manejo de contenidos o descontextualización de su uso y aplicación en la vida futura del estudiante (Vega Vega, Niño Duarte, \& Cárdena, 2015).

La siguiente sentencia parecería ser determinante "la matemática como disciplina científica está típicamente catalogada como un área de difícil comprensión, exclusiva para aquellos estudiantes que manifiestan extraordinarios talentos intelectuales" (Vílchez Quesada \& Ávila Herrera, 2021, pág. 3) esto dejaría entrever que muy pocos son los privilegiados en adentrase en el mundo de la disciplina, sin embargo, absolutamente todas las formaciones profesionales cuentan con, por lo menos, un semestre de matemáticas en sus mallas curriculares, lo que significa que nadie se escapa de su conocimiento y aprobación para avanzar en su profesión.

El conocimiento de la asignatura de matemáticas está presente desde los primeros años de formación escolar, se continúa con el bachillerato y se profundiza en la universidad, esta particularidad está presente en todos los diseños curriculares de los sistemas educativos del mundo, pero su manejo y enseñanza tiene especificidades sustanciales.

Estudios sobre el aprendizaje de las matemáticas demuestran que tienen una fuerte correlación con la comprensión lectora (Sandoval Rubilar, Frit Carrillo, Maldonado Fuentes, \& Rodríguez Alveal, 2010), lo cual tiene asidero en tanto y en cuanto una buena interpretación del problema orienta, con claridad y certeza, los mecanismos de resolución matemática aplicando el razonamiento, manejo de fórmulas, inducción y deducción, entre otras categorías intelectivas propias del pensamiento crítico matemático. 
Muchos docentes han experimentado un sinnúmero de estrategias metodológicas para potenciar la enseñanza de las matemáticas y con ello su comprensión y aprendizaje, por ejemplo, Macías Espinales (2018) usó la técnica de la gamificación para potenciar la competencia matemática "planear y resolver problemas" e incrementar la motivación por el aprendizaje de esta asignatura; al final del estudio verificó que la estrategia dio resultados importantes evidenciados a través del rendimiento académico.

Visto así, parecería ser que el aprendizaje o la enseñanza de las matemáticas es muy complicada y compleja y que dicho fenómeno se evidencia en los bajos rendimientos en esta asignatura y que, en definitiva, el estudiante no alcanza las habilidades elementales del razonamiento matemático, la resolución de problemas numéricos y solo se limita a memorizar algoritmos como mecanismo de aprendizaje. En efecto, Larrazolo, Backhoff, y Tirado (2013) realizaron un estudio en México donde corroboraron que los estudiantes tienen:

un bajo nivel de habilidades básicas de razonamiento matemático que, en teoría, se deben adquirir durante la educación básica (primaria y secundaria). Al respecto, basta señalar que los estudiantes que aspiran a ingresar al nivel de educación superior (...) presentan serias deficiencias en los aprendizajes esperados de matemáticas de los niveles de primaria y de secundaria (p. 1157).

Estos resultados solo evidencian que las debilidades del aprendizaje de las matemáticas son estructurales y se acarrea desde los niveles iniciales de formación, realidad que no es solo de México sino de muchos países, por ejemplo Ecuador, en las pruebas llevadas a cabo en el país (octubre 2017) presenta estos resultados: el 49\% de los estudiantes alcanzó el nivel mínimo de competencia en lectura, el $29 \%$ en matemáticas y el $43 \%$ en ciencias; las niñas tienen 8 puntos más que los niños en lectura, mientras que en matemáticas, los niños tienen 20 puntos más que las niñas; los estudiantes con un nivel socioeconómico alto son 3,2 veces más probables de alcanzar, por lo menos, el nivel 2 en matemáticas; el $10 \%$ de los estudiantes con mejor desempeño en Ecuador está por debajo del promedio de la OCDE en PISA 2015 en matemáticas, y en torno al promedio de América Latina (Instituto Nacional de Evaluación Educativa, INEVAL, 2018b).

Un factor determinante para mejorar la aceptación, involucramiento y rendimiento académico está relacionado con la motivación, factor intrínseco del ser humano que requiere una activación por parte del docente, "los estudiantes deben desarrollar la comprensión de los conceptos y procedimientos matemáticos y deben estar en capacidad de ver y creer que las matemáticas hacen sentido y que son útiles para ellos. Maestros y estudiantes deben reconocer que la habilidad matemática es parte normal de la habilidad mental de todas las personas, no solamente de unos pocos dotados" (Farías \& Pérez, 2010, pág. 38).

Investigaciones han demostrado que "cuanto más auto-determinado, auto-eficaz, autoregulado y espiritual sea el joven, cuanto más se involucre pro-socialmente con sus compañeros y maestros, y posea una identidad positiva y una habilidad cognitiva, mayor será el promedio que obtenga" (Barrios Gaxiola \& Frías Armenta, 2016, pág. 77). 
En definitiva, enseñar matemáticas presenta cierta complejidad, asimismo otorga al docente una oportunidad para fortalecer en el educando el interés por este campo de las ciencias y generar una alternativa de aprendizaje a lo largo de la vida.

El estudio no pretende menospreciar el currículo propuesto por el Ministerio de Educación del Ecuador, pero sí realizar una comparativa de la factibilidad de este currículo con relación a estándares internacionales como es el Bachillerato Internacional y comparar ambos currículos con un estandar evaluativo como son las pruebas PISA.

\section{Metodologia.}

Se trata de una investigación de corte cualitativo desde el paradigma interpretativo basado en la hermenéutica, su finalidad principal es contrastar los documentos oficiales del diseño curricular, tanto del bachillerato general unificado del Ecuador y el bachillerato internacional, establecido en el país desde el 2010. Por su alcance es descriptiva, transversal, no experimental y con muestreo no probabilístico intencional, puesto que de todas las áreas se escogió, deliberadamente, solo matemáticas del BGU como referente para el análisis y, aplicaciones e interpretaciones nivel superior para el BI.

El abordaje hermenéutico radica en que intenta "comprender el todo, comprender la parte y el elemento y, más en general, es preciso que texto y objeto interpretado, y sujeto interpretante, pertenezcan a un mismo ámbito, de una manera que se podría calificar de circular a la comprensión" (Arráez, Calles, \& Moreno de Tovar, 2006, pág. 171). Básicamente el trabajo operativo consistió en analizar, de principio a fin, las unidades temáticas del currículo de la asignatura de matemáticas para el BGU (Ministerio de Educación, 2016a) y para el BI (International Baccalaureate Organization, 2019), contrastar la secuencia sistemática entre unidades, el tiempo destinado al tratamiento del mismo, la complejidad de los resultados de aprendizaje y el dominio de las destrezas alcanzadas.

El trabajo está centrado en el análisis de contenidos (y de estructura) como bien lo expresa López Noguero (2002), es decir, que "se mueve entre dos polos: el del rigor de la objetividad y el de la fecundidad de la subjetividad" (p. 173). Para sistematizar la información se acogió la rigurosidad y secuencia de Tinto Arandes (2013) quien sugiere entre otros los siguientes pasos:

$\checkmark$ Primera fase, (Objetivos e hipótesis de la investigación)

$\checkmark$ Segunda fase, (Identificación del material objeto de estudio)

$\checkmark$ Tercera fase, (Definición temporal del estudio y de la unidad de análisis)

$\checkmark$ Cuarta fase, (Definición de las categorías de contenido a analizar)

$\checkmark$ Quinta fase, (Sistema de codificación para evaluar las unidades de análisis)

$\checkmark$ Sexta fase, (Codificación de la información en las unidades de análisis)

$\checkmark$ Séptima fase, (Inferencias y análisis de los datos)

$\checkmark$ Octava fase, (Presentación e interpretación de los resultados) 
La interpretación de los datos toma en consideración también los procesos de evaluación basados en las pruebas PISA para el contraste entre lo que se dicta en el desarrollo de la asignatura y lo que se evalúa internacionalmente, esto constituye un termómetro orientador para conocer si se desarrolla los contenidos en función de las exigencias nacionales e internacionales.

\section{Resultados.}

La educación es un paradigma que envuelve a una nación completa, desde sus máximos líderes hasta la última persona perteneciente al pueblo. "La educación no es preparación para la vida; la educación es la vida en sí misma” John Dewey (1859-1952). El Ecuador no está ajeno a este paradigma y constantemente está en busca de la mejora en los procesos educativos. El órgano rector de la educación en el Ecuador es el Ministerio de Educación. Este se encarga de diseñar, evaluar y reestructurar el currículo educativo y sus componentes para todos los niveles de educación. El bachillerato constituye el último escalón educativo en lo que se refiere al nivel de preparatoria, por tanto, el éxito o fracaso de un modelo educativo y su currículo se puede evidenciar en los dos últimos años de bachillerato (Ministerio de Educación, 2016b).

El currículo, al ser la estructura principal de un proyecto educativo debe estar enfocado a cubrir las principales necesidades de educación de un país. Según Herrera Pavo y Cochancela Patiño (2020) el currículo es considerado todo un proyecto pedagógico con amplitud de cobertura y complejidad sistémica donde se materializa la concepción del ser, de la realidad circundante y la naturaleza del encuentro humano. La construcción de este proyecto pedagógico va más allá del aprendizaje y se caracteriza por buscar la formación integral del ser.

El Bachillerato Internacional es una organización que busca romper las barreras territoriales de la educación moderna y enfocar el proceso enseñanza aprendizaje a una mentalidad global, sin dejar de lado el respeto a la cultura de los países que forman parte de esta organización (International Baccalaureate Organization, 2010). El Programa del Diploma (PD) es el principal ejemplo de una adaptación global de la educación y según Mayer y Perozzo-Ramírez (2020), "Este constituye una malla para los últimos dos años de la escuela secundaria que, una vez aprobado, tiene reconocimiento universal, permitiendo el ingreso automático a una diversidad de universidades en el mundo" (p. 66). Al ser de reconocimiento universal se puede aseverar de que su currículo consta de un sólido grupo de asignaturas que fortalezcan la educación de cualquier país.

Las matemáticas, entonces son parte del núcleo curricular de cualquier modelo educativo a nivel mundial. Es así que esta asignatura es evaluada mundialmente por el programa para la evaluación internacional de alumnos (PISA por sus siglas en inglés). Esta prueba estandarizada evalúa exhaustivamente y con un alto rigor académico el desempeño de los estudiantes de los países asociados a la Organización para la Cooperación y Desarrollo Económico (OCDE). Este resultado permite a esta organización recopilar datos sobre el proceso educativo en dicho país. Entre estos datos recopilados en esta evaluación se puede encontrar el currículo educativo, teniendo así una valoración general tanto de los 
objetivos, destrezas e indicadores de logro que el currículo académico alberga en su interior.

El enfoque que le da al currículo el Ministerio de Educación con relación a la asignatura de matemáticas es "La enseñanza de la Matemática tiene como propósito fundamental desarrollar la capacidad para pensar, razonar, comunicar, aplicar y valorar las relaciones entre las ideas y los fenómenos reales" (Ministerio de Educación, 2019). Esta definición permite relacionar a las matemáticas como una aplicación de lo teórico con lo práctico, además da a entender que este currículo está enfocado en cubrir las necesidades básicas para la generalidad de estudiantes del Ecuador. Esto se da debido a la reforma a la educación en el Ecuador con el Acuerdo Ministerial No. 242-11, el mismo que dicta eliminar el enfoque educativo por especialidades y cubrir las necesidades generales de la población en función de su edad. Dando así el paso para que, en los últimos tres años de educación secundaria, se dé el perfil del Bachillerato General Unificado (BGU) (Ministerio de Educación, 2011).

Analizando más a fondo el currículo del BGU se puede encontrar ciertas particularidades tanto en sus objetivos, bloques curriculares, su perfil de salida, destrezas fundamentales e indispensables y sus indicadores de logro. Junto a estas variables se analiza la carga horaria que el docente de matemáticas tiene en los tres años de estudio y compararlos con la carga de contenidos que presenta este currículo. Al final se podrá tener una perspectiva sobre este currículo y sus particularidades en el nivel de bachillerato. Previo al análisis de este es necesario aclarar los términos que engloban al documento. Matemáticas (M); Nivel Bachillerato (5); Objetivo General (OG); Criterios de Evaluación (CE) e Indicador de evaluación (IE).

Para analizar el currículo de matemática de una mejor manera es importante tomar como punto de partida los objetivos del mismo. El currículo vigente de matemáticas para la sección de bachillerato cuenta con 6 objetivos generales (OG). Estos van enfocados al desarrollo de habilidades y destrezas que contribuyen al perfil de salida del bachiller ecuatoriano. Estos objetivos son (Ministerio de Educación, 2019):

OG.M.1. Proponer soluciones creativas a situaciones concretas de la realidad nacional y mundial mediante la aplicación de las operaciones básicas de los diferentes conjuntos numéricos, y el uso de modelos funcionales, algoritmos apropiados, estrategias y métodos formales y no formales de razonamiento matemático, que lleven a juzgar con responsabilidad la validez de procedimientos y los resultados en un contexto.

OG.M.2. Producir, comunicar y generalizar información, de manera escrita, verbal, simbólica, gráfica y/o tecnológica, mediante la aplicación de conocimientos matemáticos y el manejo organizado, responsable y honesto de las fuentes de datos, para así comprender otras disciplinas, entender las necesidades y potencialidades de nuestro país, y tomar decisiones con responsabilidad social. OG.M.3. Desarrollar estrategias individuales y grupales que permitan un cálculo mental y escrito, exacto o estimado; y la capacidad de interpretación y solución 
de situaciones problémicas del medio.

OG.M.4. Valorar el empleo de las TIC para realizar cálculos y resolver, de manera razonada y crítica, problemas de la realidad nacional, argumentando la pertinencia de los métodos utilizados y juzgando la validez de los resultados.

OG.M.5. Valorar, sobre la base de un pensamiento crítico, creativo, reflexivo y lógico, la vinculación de los conocimientos matemáticos con los de otras disciplinas científicas y los saberes ancestrales, para así plantear soluciones a problemas de la realidad y contribuir al desarrollo del entorno social, natural y cultural.

OG.M.6. Desarrollar la curiosidad y la creatividad a través del uso de herramientas matemáticas al momento de enfrentar y solucionar problemas de la realidad nacional, demostrando actitudes de orden, perseverancia y capacidades de investigación.

Decodificando OG.M. se puede entender que es el objetivo general (OG), pertinente a la asignatura de matemáticas (M). Desde la perspectiva académica los objetivos descritos enfocan una visión en la que el estudiante tiene la habilidad de analizar, reflexionar críticamente y resolver lógicamente eventos de la vida real. Bajo la percepción de estos objetivos se tiene una visión del panorama educativo en el Ecuador ya que ligado a estas habilidades se encuentra la integración de las TICs y la interdisciplinariedad con otras asignaturas del currículo. En conclusión, la estructura de los objetivos para el currículo de BGU buscan conseguir un proceso de enseñanza aprendizaje ligado a que los estudiantes apliquen la teoría a la realidad de la sociedad en la que viven. Cabe mencionar que las consecuciones de estos objetivos se construyen desde los niveles inferiores de escolaridad, sin embargo, el currículo propone que los estudiantes, en los niveles de bachillerato, deben tener como perfil de salida de la escolarización general obligatoria todos estos objetivos cumplidos.

Para relacionar estos objetivos con las destrezas y los indicadores de logros de currículo de matemáticas es importante mencionar los bloques curriculares. Estos bloques están diseñados con la finalidad de organizar las destrezas y relacionarlas con el cumplimiento de los objetivos de la asignatura. Para el currículo vigente en el Ecuador, el Ministerio de Educación ha distribuido las destrezas en tres bloques: el de álgebra y funciones, geometría y medida y finalmente el de probabilidad y estadística. La idea de compactar los contenidos pedagógicos en tres bloques se da por la afinidad y correlación entre los temas principales y los contenidos previos necesarios para abordar los mismos.

Cada uno de estos bloques temáticos van desglosando contenidos que, posteriormente, serán desarrollados con las destrezas con criterio de desempeño; para evaluar cada bloque curricular es importante detallar los contenidos de cada uno con la finalidad de comparar la cantidad de destrezas necesarias para cubrir cada bloque.

\section{Bloque de algebra y funciones}

En este primer bloque se tiene 7 temas generales de trabajo. Hay que tener en cuenta que muchos de estos temas tienen implícitas en el contenido temático operaciones y 
propiedades necesarias para su análisis. Entonces, fácilmente estos temas pueden llegar a desglosarse con mayor profundidad en aproximadamente 45 subtemas. Distribuyéndolos en los tres años de estudio, el estudiante de bachillerato, únicamente para el primer bloque, debe cubrir como mínimo 15 subtemas. Teniendo en cuenta el perfil de salida y los objetivos curriculares, el estudiante en estos temas debe, como mínimo, conocer los principios de modelamiento matemático (OG.M.1), la resolución de problemas (OG.M.2), el uso de las TICS (OG.M.3).

\section{Bloque de geometría y trigonometría}

En este segundo bloque constan nuevamente 7 temas de trabajo con los estudiantes. Estos temas deben ser cubiertos en los tres años de bachillerato, sin embargo, cada tema necesita ser desglosado en subtemas de estudio. En una perspectiva general cada tema como mínimo aborda 3 subtemas de desarrollo, entonces se puede tener en cuenta que para este segundo bloque será necesario sumar a la carga de contenidos 21 subtemas a ser abordados. Es decir, se deberán trabajar con 7 subtemas de este bloque en cada año. Junto a estos temas aportan a la consecución de los objetivos y el perfil de salida en relación a las construcciones geométricas (OG.M.4).

\section{Bloque de estadística y probabilidad.}

Para el último bloque de estudio se cubren 4 temas para ser abordados en los tres años de estudio. Sin embargo, los subtemas relacionados a este bloque son alrededor de 6 por cada tema general. Es decir, se debe cubrir como mínimo 8 subtemas en cada año para abordar los 24 subtemas del bloque. Añadiendo esta cantidad temática, a la ya cargada valoración de los bloques anteriores, se puede entonces tener una primera idea de las dificultades que el docente y el alumno tienen al cumplir con los objetivos y los criterios de evaluación del currículo académico propuesto.

Una vez que se ha hecho el estudio temático de cada uno de los bloques del currículo de matemáticas para el BGU, se debe analizar las destrezas con criterio de desempeño de cada bloque, con la finalidad de relacionar la cantidad de destrezas que cubren cada bloque con la carga horaria. Las destrezas con criterio de desempeño serán analizadas en valores numéricos. Esto permitirá analizar la cantidad de horas pedagógicas para la asignatura y la cantidad de destrezas a cubrir. Es necesario realizar una especificación sobre estas destrezas. El Ministerio de Educación del Ecuador propone dos tipos de destrezas con criterio de desempeño: las básicas imprescindibles, que como su nombre lo indica deben ser tratadas como obligatorias dentro de las planificaciones micro curriculares de los docentes que dictan matemáticas en el BGU; las básicas deseables, si bien es cierto no son obligatorias, se sugieren que deban incluirse las más importantes a consideración del docente en las planificaciones. 
Tabla 1

Destrezas con criterio de desempeño descritas por cada bloque curricular.

\begin{tabular}{lccc}
\hline Bloque & $\begin{array}{l}\text { Básicas } \\
\text { imprescindibles }\end{array}$ & Básicas deseables & Total \\
\hline Álgebra y funciones & 54 & 24 & 78 \\
Geometría y trigonometría & 16 & 11 & 27 \\
Estadística y probabilidades & 15 & 10 & 25 \\
Total & 85 & 45 & 130 \\
\hline
\end{tabular}

Fuente: Elaboración propia basada en la malla curricular de Matemática del MINEDUC

En la tabla 1 se puede observar que la cantidad de destrezas para abordar en los tres años de estudio son de 85 obligatorias y 45 a selección del docente. Para analizar la cantidad de temas a abordar es primordial entender la carga horaria que el currículo de matemática sugiere para cada año académico. La tabla 2 muestra la distribución de la carga horaria sugerida por el Ministerio de Educación a las instituciones educativas, independientemente del tipo de sostenimiento: fiscales, particulares y fiscomisionales. Esta carga horaria está detallada por horas a la semana. Es importante clarificar que el periodo de clase en el Ecuador está dado por 45 minutos. En relación a la cantidad de días que un año lectivo tiene en el país son 200 días laborales. Estos distribuidos en dos quimestres de trabajo cada uno con tres parciales de trabajo; en la tabla 3 se da una generalidad de los días. Debido a la pandemia producida por el covid-19 el Ministerio de Educación realizó una reestructuración del periodo académico 2020-2021. Estos cambios se dieron en la reducción de los tres parciales a dos parciales, sin embargo, para fines del presente estudio se realiza el análisis para el proceso educativo pre pandemia y post pandemia.

Tabla 2

Carga horaria semanal por año de bachillerato en la asignatura de matemáticas.

\begin{tabular}{llll}
\hline Asignatura & $\begin{array}{l}\text { 1er año de } \\
\text { Bachillerato }\end{array}$ & $\begin{array}{l}\text { 2do año de } \\
\text { Bachillerato }\end{array}$ & $\begin{array}{l}\text { 3er año de } \\
\text { Bachillerato }\end{array}$ \\
\hline Matemáticas & 5 & 4 & 3 \\
\hline Fuente: Elaboración propia basada en la malla curricular de Matemática del MINEDUC
\end{tabular}

Tabla 3

Detalle de días de trabajo y días de evaluaciones del bachillerato.

\begin{tabular}{lll}
\hline Período & Días & \multicolumn{1}{c}{ Observaciones } \\
\hline PRIMER QUIMESTRE & & \\
Parcial 1 & 100 & 1 semana de evaluación de fin \\
& & del parcial. \\
Parcial 2 & & 1 semana de evaluación de fin \\
& del parcial. \\
Parcial 3 & 1 semana de evaluación de fin \\
& del parcial y 1 semana de \\
& evaluación quimestral. \\
\hline
\end{tabular}


Tabla 3

Detalle de días de trabajo y días de evaluaciones del bachillerato. (continuación)

\begin{tabular}{|c|c|c|}
\hline Período & Días & Observaciones \\
\hline \multicolumn{3}{|c|}{ SEGUNDO QUIMESTRE } \\
\hline Parcial 1 & 100 & $\begin{array}{l}1 \text { semana de evaluación de fin } \\
\text { del parcial. }\end{array}$ \\
\hline Parcial 2 & & $\begin{array}{l}1 \text { semana de evaluación de fin } \\
\text { del parcial. }\end{array}$ \\
\hline Parcial 3 & & $\begin{array}{l}1 \text { semana de evaluación de fin } \\
\text { del parcial y } 1 \text { semana de } \\
\text { evaluación quimestral. }\end{array}$ \\
\hline
\end{tabular}

Fuente: Elaboración propia basada en la malla curricular de Matemática del MINEDUC

En la tabla 3, según las observaciones dadas, los 200 días de trabajo se ven reducidos a 160 días de actividad en aula. Los 40 días previstos para las evaluaciones, los docentes no pueden tratar destrezas nuevas, sino que se sugiere se realicen refuerzos académicos previo a las evaluaciones y, posteriormente, se recomienda realizar el solucionario de las mismas, es decir, que el docente del nivel de bachillerato tiene para trabajar, dependiendo el nivel, 5 horas, 4 horas o 3 horas a la semana. La tabla 4 muestra la cantidad de horas clase en cada año de bachillerato en relación a la cantidad de días del año escolar.

Tabla 4

Horas de clase por año de BGU

\begin{tabular}{lc}
\hline Año & Horas clase \\
\hline 1er año de bachillerato & 160 \\
2do año de bachillerato & 120 \\
3er año de bachillerato & 80 \\
Total & 360 \\
\hline
\end{tabular}

Fuente: Elaboración propia basada en la malla curricular de Matemática del MINEDUC

Tal como se menciona en la tabla 4, el docente de bachillerato cuenta con 360 horas de clase durante los tres años de estudio para cubrir una temática de 85 destrezas básicas imprescindibles y 45 destrezas básicas deseables. Previo a realizar un análisis comparativo de la cantidad de horas a trabajar en cada destreza se debe puntualizar que el Ministerio de Educación sugiere que se tomen como horas de imprevistos 10 horas. Estas 10 horas se enfocan en supuestos sucesos fuera del plan de feriados nacionales y se enfocan en cubrir posibles horas de clases no dictadas ocasionadas por eventos de la institución fuera del plan de trabajo anual. Bajo este análisis, se puede afirmar que en las 350 horas de trabajo el docente de bachillerato debe abordar los temas y destrezas propuestos. En la tabla 5 se muestran las posibles combinaciones y el número de horas por destreza que el docente tendrá para lograr que los estudiantes dominen la misma. 
Tabla 5

Relación entre destrezas, horas clase y horas reloj

\begin{tabular}{|c|c|c|c|}
\hline $\begin{array}{l}\text { Combinación de } \\
\text { destrezas }\end{array}$ & $\begin{array}{l}\text { Total de } \\
\text { destrezas }\end{array}$ & $\begin{array}{l}\text { Horas académicas } \\
\text { por destreza }\end{array}$ & $\begin{array}{l}\text { Horas de trabajo por } \\
\text { destreza }\end{array}$ \\
\hline $\begin{array}{ll}\text { Solo } & \text { Básicas }\end{array}$ & 85 & $350 / 85=4,12$ & $4,12 * 45=185,4 / 60=$ \\
\hline Imprescindibles & & & 3,09 \\
\hline $\begin{array}{l}\text { Básicas imprescindibles } \\
\text { y el } 25 \% \text { de básicas } \\
\text { deseables }\end{array}$ & $85+12=97$ & 3,61 & 2,70 \\
\hline $\begin{array}{l}\text { Básicas imprescindibles } \\
\text { y el } 50 \% \text { de básicas } \\
\text { deseables }\end{array}$ & $85+23=108$ & 3,24 & 2,43 \\
\hline $\begin{array}{l}\text { Básicas imprescindibles } \\
\text { y el } 75 \% \text { de básicas } \\
\text { deseables }\end{array}$ & $85+34=119$ & 2,94 & 2,21 \\
\hline $\begin{array}{l}\text { Básicas imprescindibles } \\
\text { y básicas deseables }\end{array}$ & $85+45=130$ & 2,69 & 2,02 \\
\hline
\end{tabular}

En la tabla 5 se evidencia el tiempo que el docente dispone para cada una de las posibilidades más comunes en el proceso enseñanza aprendizaje de matemáticas. En la segunda columna se puede observar que para cubrir una destreza el docente dispone de 3,61 clases y, dependiendo del horario de trabajo, el docente tendría como máximo tres encuentros con los estudiantes para cubrir una destreza. Desde la experiencia, como docentes de matemáticas, se puede afirmar que, dentro del horario de clases, es muy común encontrarse con 2 horas seguidas de esta asignatura, como mínimo. Es decir, se tendría únicamente dos encuentros con los dicentes para cubrir una destreza. En este punto se está sujeto a la crítica en relación a que hay algunas destrezas que se pueden cubrir más rápido que otras. Si bien es cierto, en este análisis, la pregunta retórica sería, ¿El estudiante puede dominar una destreza en solo dos clases? En el proceso metodológico de una clase de matemáticas, para determinar que un estudiante ha dominado una destreza, este debe estar en la capacidad de resolver tres ejercicios seguidos de nivel medio sin errores y en un tiempo promedio de 2 minutos por problema. Entonces, en una media de 2,6 horas de clase, se puede afirmar que, probablemente, el estudiante alcance el nivel de logro básico. Aún más, es difícil asegurar que el 100\% de la clase logre llegar a este nivel teniendo en cuenta variables como la heterogeneidad de la clase, las necesidades educativas, los estilos de aprendizaje, la disposición volitiva del estudiante, entre otras.

Para evaluar estas destrezas el BGU propone criterios de evaluación (CE), estos van ligados directamente a un grupo de destrezas. Es decir, se cuenta con 11 criterios de evaluación. Estos dan al docente una dirección de lo que se espera que el estudiante pueda lograr al ser evaluado. Un ejemplo de estos criterios es CE.M.5.5. Aplica el álgebra de límites como base para el cálculo diferencial e integral, interpreta las derivadas de forma geométrica y física, y resuelve ejercicios de áreas y problemas de optimización. Como se detalla en este quinto criterio que evalúa destrezas de cálculo, el docente tiene un 
panorama puntual de lo que debe conseguir con los estudiantes en el proceso enseñanza aprendizaje.

Hasta aquí el análisis de lo que ocurre con el BGU en el currículo de matemáticas en el Ecuador. La siguiente explicación y descripción corresponde al BI que también se encuentra en el sistema educativo ecuatoriano.

El Bachillerato Internacional propone a los estudiantes dos asignaturas de matemáticas enfocado a suplir cada una de las necesidades de aprendizaje. En su política del currículo se refiere a la función de las matemáticas en explorar la naturaleza y sus fenómenos, teniendo como referente el desarrollo de habilidades en sus cinco grupos fundamentales: el álgebra, funciones, geometría y trigonometría, probabilidad y estadística y análisis (International Baccalaureate Organization, 2020). Si el estudiante desea seguir en la universidad carreras afines a las áreas humanísticas como: medicina, docencia, jurisprudencia, etc. puede optar por el módulo de Aplicaciones e interpretaciones, el mismo que tiene más enfoque en el desarrollo de la estadística. Sin embargo, si el estudiante tiene propuesto enfocar sus estudios de tercer nivel en el área de la ingeniería, el módulo que la organización recomienda es el de Análisis y enfoques, el mismo que tiene un gran desempeño y profundización en la parte del cálculo. Para el estudio comparativo con el BGU se utilizará la guía de estudio de la asignatura de Aplicaciones e Interpretaciones. Es importante hacer un paréntesis para especificar que estas dos asignaturas se dictan en dos niveles de dificultad, el Nivel Medio (NM) y el Nivel Superior (NS). Para el análisis actual se trabajó con la máxima cantidad de contenidos a abordar por parte del estudiante y del docente. Es decir, se realizó todos los cálculos con la cantidad de subtemas y horas para el Nivel Superior.

A diferencia del BGU, el Bachillerato Internacional define algunas características importantes para la orientación docente. En el caso de las destrezas el bachillerato internacional las define como habilidades; se debe resaltar que estas habilidades no son dadas por la organización, sino que le da autonomía al docente en crearlas en función de las temáticas a tratar. También es importante revisar los objetivos que propone la organización para la asignatura de matemáticas en general. Entre estos, se describen 12 objetivos que los estudiantes deben cumplir durante los dos años de diploma, y son los siguientes:

1. Desarrollar su curiosidad por las matemáticas, disfrutarlas, y apreciar su elegancia y las posibilidades que ofrecen.

2. Desarrollar una comprensión de los conceptos, los principios y la naturaleza de las matemáticas.

3. Comunicar las matemáticas con claridad, concisión y confianza en diversos contextos.

4. Desarrollar el pensamiento lógico y creativo, así como la paciencia y la constancia en la resolución de problemas, para adquirir confianza en el empleo de las matemáticas.

5. Emplear y perfeccionar sus capacidades de abstracción y generalización 
6. Dar los pasos necesarios para aplicar y transferir habilidades a distintas situaciones, a otras áreas del conocimiento y a avances futuros en sus comunidades locales y globales.

7. Apreciar cómo los avances tecnológicos influyen en los avances en matemáticas y viceversa.

8. Apreciar las cuestiones morales, sociales y éticas del trabajo de los matemáticos y las aplicaciones de las matemáticas.

9. Apreciar la universalidad de las matemáticas y sus perspectivas multiculturales, internacionales e históricas.

10. Valorar la contribución de las matemáticas a otras disciplinas y como área de conocimiento específica en el curso de TdC (Teoria del Conocimiento).

11. Desarrollar la capacidad de reflexionar de manera crítica sobre su propio trabajo y el de los demás.

12. Ampliar su comprensión de las matemáticas de manera independiente y en colaboración.

En los objetivos, se tiene una clara perspectiva de lo que el programa del diploma sugiere se trabaje en los estudiantes, generalizando estas ideas se entiende entonces que el dicente que tome la asignatura de matemáticas, independientemente a un conocimiento profundo, busca que tenga conciencia mundial, propone una idea de trasnversalidad de contenidos y finalmente relacione todos estos con el desarrollo tecnológico.

Para estudiar los contenidos que esta guía propone para los docentes y estudiantes, es primordial entender que, al igual que el Ministerio de Educación del Ecuador, el Bachillerato Internacional los ha organizado en 5 temas generales: Tema 1, Aritmética y álgebra; Tema 2, Funciones; Tema 3, Geometría y Trigonometría; Tema 4, Estadística y Probabilidad; y, Tema 5, Análisis. Es importante revisar la cantidad de subtemas que abordan cada tema, por ejemplo, para el tema 1, los estudiantes deben desarrollar habilidades para 15 subtemas; para el caso del tema 2 se observa que las habilidades requeridas son de 10; en el caso de geometría y trigonometría se tiene una cantidad de 16 subtemas a ser abordados; para el bloque 4, el estudiante deberá tener las habilidades de 19 subtemas y, finalmente, para el bloque 5, de análisis, se debe abordar 18 temas de estudio. Al contabilizar todos los subtemas de los 5 bloques, se tienen 78 subtemas que se deben cubrir en un tiempo de dos años.

En cuanto a la carga horaria de la asignatura en la guía de estudios el Bachillerato Internacional propone una clara estructura de carga horaria lectiva por tema. En la tabla 6 se tiene una visión panoramica de cada tema y la cantidad de horas lectivas que el docente tiene para poder trabajar en la misma. Se debe aclarar que esta cantidad de horas sugeridas se dan para los dos años del diploma.

Tabla 6

Horas lectivas sugeridas por el IBO para cada tema

\begin{tabular}{lcc}
\hline Tema & Cantidad de horas lectivas \\
\hline Tema 1: Aritmética y álgebra & 29 & \\
\hline & & \\
\hline Desarrollo & Página $\mathbf{6 2}$
\end{tabular}


Horas lectivas sugeridas por el IBO para cada tema (continuación)

\begin{tabular}{lc}
\hline Tema & Cantidad de horas lectivas \\
\hline Tema 2: Funciones & 42 \\
Tema 3: Geometría y trigonometría & 46 \\
Tema 4: Estadística y probabilidad & 52 \\
Tema 5: Análisis & 41 \\
Total & 210
\end{tabular}

Fuente: Elaboración propia basada en la malla curricular de Matemática del BI

En la tabla 6 se tiene una primera visión de la cantidad de horas académicas que el docente debe enfocar para cubrir cada tema. Haciendo una relación con la cantidad de horas académicas en el BGU se ve una gran diferencia en carga horaria y de contenidos. En el BGU tenemos 130 destrezas a trabajar en una carga horaria de 360 horas. Para el caso del BI la relación se da en 78 subtemas con una carga horaria de 420 horas lectivas. En la tabla 7 se da una perspectiva más clara de la relación de subtemas y carga horaria en el Bachillerato Internacional. Es importante mencionar que las horas académicas que el BI propone son de 60 minutos.

Tabla 7

Análisis de cantitad temática y horas recomendadas.

\begin{tabular}{lcc}
\hline Temas & Total de habilidades & $\begin{array}{c}\text { Horas académicas por } \\
\text { habilidad. }\end{array}$ \\
\hline Tema 1: Aritmética y Álgebra & 15 & $29 / 15=1,93$ \\
Tema 2: Funciones & 10 & 4,2 \\
Tema 3: Geometría y trigonometría & 16 & 2,88 \\
Tema 4: Estadística y probabilidad & 19 & 2,74 \\
Tema 5: Análisis & 18 & 2,28 \\
\hline
\end{tabular}

Fuente: Elaboración propia basada en la malla curricular de Matemática del BI

En el análisis por tema, se puede observar que la cantidad más baja de horas de trabajo por subtema se da en el primer bloque de Aritmética y Álgebra. Sin embargo, al hacer una revisión a fondo de los subtemas y, tomando en cuenta la experiencia docente al tratar los mismos, la cantidad de horas que se distribuyen para estos contenidos son manejables. A diferencia de las analizadas en el caso de la carga horaria por destreza en el BGU se tiene todas las destrezas enmarcadas en un mismo tiempo promedio. En el caso del BI la claridad al momento de orientar la información permite que el docente tenga un norte en el proceso enseñanza aprendizaje. También es importante comprender, como se menciona en párrafos anteriores, que esta asignatura está enfocada para los estudiantes que tienen una meta de tercer nivel en el área de las ciencias humanísticas. Por esta razón la carga horaria que se tiene en el bloque 4 es bastante alta, y aunque no tiene mayor enfoque para la rama de las ingenierías es sorprendente poder observar que el tema 5 tiene una carga de contenidos bastante elevada. Esto lleva a una primera conclusión preliminar que, aunque esta asignatura supone un apoyo para que los estudiantes puedan continuar sus estudios superiores en carreras en ciencias humanas, también brinda la factibilidad de que un estudiante de esta asignatura siga una carrera de ingeniería, dicho escenario no está 
muy alejado de la realidad. Esta visión del BI permite entonces entender el enfoque que el Ministerio de Educación quiere dar al BGU al proveer al futuro bachiller la posiblidad de elegir la carrera de su preferencia.

Para el caso de la evaluación de estas habilidades, el BI maneja una disposición diferente con relación a los criterios de evaluación del Ministerio de Educación. Es así que la organización pretende que el proceso de evaluación se vea enfocada en cumplir los objetivos de evaluación. La guía del BI entonces propone que el estudiante deba cumplir con 6 objetivos de evaluación. Lo interesante de estos objetivos se da en que no están orientados para un grupo de habilidades como en el caso del BGU, sino que el docente, durante todo el proceso enseñanza aprendizaje debe ir cumpliendo con estos objetivos generales que se detallan a continuación:

1. Conocimiento y comprensión: recordar, seleccionar y utilizar su conocimiento de las técnicas, los hechos y los conceptos matemáticos en una diversidad de contextos conocidos y desconocidos.

2. Resolución de problemas: recordar, seleccionar y utilizar su conocimiento de las habilidades, los resultados y los modelos matemáticos, tanto en contextos abstractos como reales, para resolver problemas.

3. Comunicación e interpretación: transformar en matemáticas contextos realistas comunes; hacer comentarios sobre el contexto; dibujar aproximadamente o con precisión diagramas, construcciones o gráficos matemáticos, tanto en papel como utilizando medios tecnológicos; registrar métodos, soluciones y conclusiones utilizando notación estandarizada; utilizar notación y terminología apropiada.

4. Tecnología: utilizar los medios tecnológicos de forma precisa, adecuada y eficaz para explorar nuevas ideas y resolver problemas.

5. Razonamiento: elaborar argumentos matemáticos mediante el uso de enunciados precisos,

deducciones lógicas e inferencia, y mediante la manipulación de expresiones matemáticas.

6. Enfoques basados en la indagación: investigar situaciones desconocidas, tanto abstractas como reales, que conllevan la organización y el análisis de información, la formulación de conjeturas, la extracción de conclusiones y la comprobación de su validez.

La característica que brindan estos objetivos de evaluación al docente radica en que el aprendizaje es integral y van directamente relacionados con los objetivos de la asignatura. La forma en combinar el conocimiento con la forma de expresar y comunicar sus ideas utilizando medios tecnológicos, dando respuestas razonadas en función de la indagación, hacen que el alumno tenga una metacognición completa del entorno de las matemáticas.

Ahora, si bien es cierto, se observa una clara ventaja en el trabajo en aula del BI con relación al BGU. No se tiene un veredicto final sobre cuál de los dos currículos educativos es más objetivo y beneficia de mejor manera al estudiante. Si bien es cierto se tiene una cantidad relativamente más baja en el BI, no es una garantía de que el programa abarque 
mejores resultados. Entonces, para aclarar esta nueva incógnita resulta factible hacer un análisis proporcional entre la cantidad de temas y subtemas en cada uno de los programas de estudio y una evaluación estandarizada que permita observar cuál de los dos es más útil al momento de resolver problemas. Para este análisis se va a tomar en cuenta un ejemplo de evaluación PISA.

Las pruebas PISA son completamente confidenciales, por tanto, cada cierto tiempo la OCDE libera un modelo con la finalidad de que los países miembros de la organización puedan comparar sus actividades en clase con las propuestas en la evaluación. En una revisión breve de la evaluación PISA se puede notar los siguientes temas: relaciones, conversión de unidades, análisis espacial, histogramas, progresiones aritméticas y geométricas, cálculo, estadística descriptíva, interpretación de datos en gráficas, áreas y perímetros, ecuaciones, tasa de cambio, probabilidad simple y condicional, sistemas de ecuaciones con dos y tres incógnitas, proporciones, combinaciones.

Es preciso revisar la cantidad de veces que se repite cada tema y relacionarlos con las horas que un estudiante trabaja en el mismo, por tanto, será la clave fundamental para estudiar su utilidad frente al momento en la que el estudiante se encuentra con la resolución de problemas reales. La tabla 8 muestra la cantidad de temas tratados en las pruebas PISA liberadas y la cantidad de horas en cada tipo de bachillerato en el Ecuador (Instituto Nacional de Evaluación y Calidad del Sistema Educativo, [INECSE], 2005).

Tabla 8

Relaciones entre preguntas PISA y horas de trabajo en Bachillerato.

\begin{tabular}{lccc}
\hline Tema & $\begin{array}{c}\text { Cantidad de } \\
\text { preguntas }\end{array}$ & $\begin{array}{c}\text { Cantidad de } \\
\text { horas al BGU }\end{array}$ & $\begin{array}{c}\text { Cantidad de } \\
\text { horas al BI }\end{array}$ \\
\hline Relaciones & 5 & 2,05 & 1,93 \\
Conversión de unidades & 3 & 2,05 & 1,93 \\
Análisis espacial & 4 & 4,44 & 2,88 \\
Histogramas & 4 & 3,2 & 2,74 \\
Progresiones aritméticas y geométricas & 1 & 2,05 & 1,93 \\
Cálculo & 2 & 2,05 & 4,2 \\
Estadística descriptíva & 5 & 3,2 & 2,74 \\
Interpretación de datos en gráficas & 5 & 2,05 & 1,93 \\
Áreas y perímetros & 2 & 4,44 & 2,88 \\
Ecuaciones & 1 & 2,05 & 4,2 \\
Tasa de cambio & 2 & 2,05 & 1,93 \\
Probabilidad simple y condicional & 1 & 3,2 & 2,74 \\
Sistemas de ecuaciones con dos y tres incógnitas & 1 & 2,05 & 4,2 \\
Proporciones & 3 & 2,05 & 1,93 \\
Combinaciones & 1 & 2,05 & 1,93 \\
\hline
\end{tabular}

Fuente: Elaboración propia basada en las pruebas PISA

Al analizar estadísticamente esta tabla, es importante considerar los siguientes parámetros: se observan 40 preguntas que el estudiante debe desarrollarlas en un determinado tiempo de la evaluación; se establece que para realizar esta actividad el estudiante estuvo en contacto con alrededor de 40,09 horas, para el caso del BI; y, para el 
BGU, al no tener una estructura de clase de distribución temática, se procede a trabajar con el supuesto de que en cada año se cubre un bloque de aprendizaje, con la finalidad de tener un escenario bueno donde se relacione la mayor cantidad de destrezas con la mayor cantidad de horas. En este caso, para el BGU el estudiante tiene 38,98 horas para trabajar con los temas previstos para solucionar esta evaluación. En este punto se puede tener una primera idea de que en el BI al tener una estructura más clara de temas y cantidad de subtemas, el docente tiene una mejor distribución de tiempo, entonces tiene mayor espacio para cubrir las habilidades deseadas en las pruebas PISA. En el caso del BGU al tener un solo bloque con mayor cantidad de contenidos se prevee que el docente tendrá menor tiempo para trabajar en función a esta prueba. Si se hace una relación entre preguntas y tiempo está claro que la proporción para el BI es de 0,998, mientras que para el BGU existe una proporción de 1,027. Entendiéndolo de mejor manera el estudiante de BI resolverá una pregunta en menor tiempo con relación al estudiante de BGU.

En el Ecuador existen alrededor de 16102 unidades educativas según el último senso por parte del MINEDUC (Ministerio de Educación, 2021). En relación a los colegios que tienen BI según el ASECCBI (Asociación Ecuatoriana de Colegio de Bachillerato Internacional) en el país existen 236 colegios, es decir, solo $0,065 \%$ de los colegios dictan este programa. Según los datos recabados, se puede tener una primera idea de que la educación del BI, si bien es cierto, no supera altamente a la del BGU, si se observa un mejor aporte al conocimiento de los estudiantes. Al tener una estructura más clara, el docente es capaz de organizar mejor el tiempo, de igual manera, al tener una cantidad de temas menor, el BI tiene más tiempo de fortalecer las habilidades.

\section{Conclusiones.}

- El objetivo general del BGU busca que el estudiante pueda dar solución a los problemas que se le presenten en su realidad, utilizando los medios y herramientas tecnológicos, tal cual como se muestran en los objetivos de evaluación detallados en el documento.

- En el caso del BI se nota una mentalidad más abierta a resolver estos mismos problemas, no solo enfocando su estrategia en solucionar los mismos por medios tecnológicos, sino que sea capaz de dar una solución con mentalidad global. Surge la incertidumbre de si la educación debe estar enfocada a la realidad nacional o a la realidad mundial. Cabe aclarar que las condiciones sociales, económicas y culturales son importantes para que el educador pueda desarrollar estas destrezas o habilidades en el estudiantado, tambien es menester responsabilizar al docente en que la realidad global es mucho más importante que la realidad nacional, no restando valor a los saberes ancestrales, sino pretendiendo que la educación se base en el cuidado del entorno de convivencia mundial. La educación no tiene brecha fronteriza, debe ser por tanto, abierta y enfocada a resolver los problemas tanto globales como regionales.

- No fue sorprendente encontrar que la cantidad de temas en el BGU son mucho mayores al currículo previsto por el BI, de la misma manera, la forma en la que el currículo del BGU propone al docente los temas a cubrir durante los tres años de 
estudio pone en duda la "flexibilidad" que propone el enunciado de este documento. Si bien es cierto proponer un listado de temas ayuda al docente a preparar su plan anual de trabajo, la excesiva lista hace que el docente se vea obligado a reducir el tiempo de ciertas destrezas con la finalidad de cubrir "lo mínimo" que el MINEDUC propone.

- Ante esta realidad es de vital importancia realizar un estudio de las destrezas imprescindibles y de las básicas deseadas, no con el objetivo de eliminar "las que no sirven", ya que dentro de la educación es importante tener mayor conocimiento, sino que se reestructure posiblemente descendiendo algunas destrezas a niveles inferiores o a su vez con un equipo de catedráticos unir algunas de ellas con otras que tiene similitud, logrando así que el docente potencie una destreza que desarolle varios campos cognitivos.

- Finalmente, la relación entre la cantidad de temas a evaluar en una prueba estandarizada y los diferentes tipos de bachillerato deja a la reflexión del lector la importancia de enfocar los esfuerzos en el aula, no a cubrir temas, tampoco desarrollar habilidades o destrezas, sino que la educación debe enfocarse a resolver los problemas. Muy repetidamente se ha escuchado la frase en los estudiantes "y esto para que me sirve", si el estudiante no aprende a relacionar lo teórico con lo práctico, estas frases serán las comunes en los salones de clase.

- Las evaluaciones internacionales no pretenden que el estudiante demuestre sus conocimientos al pie de la letra, por el contrario, buscan que el estudiantado aplique varios conocimientos, que relacionados con su experiencia en la realidad, puedan obtener una solución óptima. Si el docente de matemáticas se dedica a que el texto, el ejercicio, la actividad, sea similar a lo que se trabaja en clase, es muy probable que los resultados se asemejen a los del año 2018, pero si el docente deja a un lado estas herramientas y presenta un problema en el que los antiguos conocimientos se relacionen con el nuevo conocimiento, no solo enfocará su enseñanza a que la resolución de las pruebas PISA mejore, sino que tambien promoverá, en el jóven, la curiosidad por cambiar el "y esto para que me sirve" a ¿Cuánto puedo solucionar con lo que aprendí?\}

\section{Referencias bibliográficas.}

Arévalo Duarte, M. A., \& Gamboa Suárez, A. A. (enero-junio de 2015). TIC en el curriculo de matemáticas. Una orientación desde el marco de las politicas y proyectos educativos. Revista Interamericana de Investigación, Educación y Pedagogía, 8(1), 169-187. Recuperado el 16 de mayo de 2021, de http://www.redalyc.org/articulo.oa?id=561058728006

Arráez, M., Calles, J., \& Moreno de Tovar, L. (2006). La Hermenéutica:una actividad interpretativa. Sapiens. Revista Universitaria de Investigación, 7(2), 171-181. Recuperado el 2 de junio de 2021, de https://www.redalyc.org/pdf/410/41070212.pdf 
Barrios Gaxiola, M. I., \& Frías Armenta, M. (2016). Factores que influyen en el desarrollo y rendimiento escolar de los jóvenes de Bachillerato. Reviscta colombiana de psicología, 25(1), 63-82. Recuperado el 27 de mayo de 2021, de 10.15446/rcp.v25n1.46921

de Zubiría Samper, J. (2013). ¿Cómo diseñar un currículo por competencias? Fundamentos, lineamientos y estrategias. Bogotá DC., Colombia: Magisterio Editorial. Recuperado el 17 de mayo de 2021

Farías, D., \& Pérez, J. (2010). Motivación en la enseñanza de las Matemáticas y la Administración. Formación Universitaria, 3(6), 33-40. doi:10.4067/S071850062010000600005

Herrera Pavo, M. Á., \& Cochancela Patiño, M. G. (2020). Aportes de las reformas curriculares a la educación obligatoria en el Ecuador. Revista Scientific, 5(15), 362-383. doi:https://doi.org/10.29394/Scientific.issn.2542-

2987.2020.5.15.19.362-383

Instituto Nacional de Evaluación Educativa, INEVAL. (octubre de 2018a). Estudio comparativo de los resultados de Ser Bachiller 2017 para estudiantes de Bachillerato Internacional Vs. los de Bachillerato General Unificado en Ciencias. Quito, Ecuador: INEVAL. Recuperado el 17 de mayo de 2021, de http://www.evaluacion.gob.ec/wpcontent/uploads/downloads/2019/01/DIED_BIvsBGUC_20190123.pdf

Instituto Nacional de Evaluación Educativa, INEVAL. (2018b). Educación en Ecuador. Resultados PISA para el Desarrollo. Quito, Ecuador: Instituto Nacional de Evaluación Educativa. Recuperado el 27 de mayo de 2021, de http://evaluaciones.evaluacion.gob.ec/BI/download/907/

Instituto Nacional de Evaluación y Calidad del Sistema Educativo, [INECSE]. (2005). PISA 2003. Pruebas de Matemáticas y solución de problemas. Madrid: Misterio de Educación y Ciencias. Obtenido de http://pisaparacentroseducativos.es/pdf/Items\%20liberados\%20Matem\%C3\%A1 ticas.pdf

International Baccalaureate Organization. (2010). Bachillerato Internacional. Programa del Diploma. Recuperado el 30 de 03 de 2021, de https://ibo.org/es/programmes/diploma-programme/

International Baccalaureate Organization. (2014). Programa de los Años Intermedios. Ginebra, Suiza: Organización del Bachillerato Internacional. Recuperado el 17 de mayo de 2021

International Baccalaureate Organization. (2019). ¿Qué es la educación del IB? Ginebra, Suiza: Organización del Bachillerato Internacional. Recuperado el 17 de mayo de 2021, de https://www.ibo.org/globalassets/what-is-an-ib-education-es.pdf 
International Baccalaureate Organization. (2020). Guía de Matemáticas: Aplicación e interpretación. Ginebra: International Baccalaureate Organization. Recuperado el 3 de junio de 2021, de https://xmltwo.ibo.org/publications/EAD/2021_SP/GR5/GR5-Maths_AI_SP.pdf

Larrazolo, N., Backhoff, E., \& Tirado, F. (2013). Habilidades de razonamiento matemático de estudiantes de educación media superior en México. Revista Mexicana de Investigación Educativa, 18(59), 1137-1163. Recuperado el 27 de mayo de 2021, de http://www.redalyc.org/articulo.oa?id=14028945006

López Noguero, F. (2002). El análisis de contenido como método de investigación. Revista de Educación XXI, 4, 167-179. Recuperado el 2 de junio de 2021, de http://rabida.uhu.es/dspace/bitstream/handle/10272/1912/b15150434.pdf;El

Macías Espinales, A. (2018). Gamificación en el desarrollo de la competencia matemática: Plantear y Resolver Problemas. Sinapsis, 12(1), 1-18. Recuperado el 27 mayo de $2021, \quad$ de https://revistas.itsup.edu.ec/index.php/sinapsis/article/download/136/127/693

Malagón Plata, L. A., Rodríguez Rodríguez, L. H., \& Ñáñez Rodríguez, J. J. (2019). El currículo: fundamentos teóricos y prácticos (Primera ed.). Ibagué: Universida de Tolima. Recuperado el 16 de mayo de 2021, de http://repository.ut.edu.co/bitstream/001/3023/2/El_Curri\%CC\%81culo\%2CFun damentos_Teoricos_Contenido_10_09_2019.pdf

Mayer, L., \& Perozzo-Ramírez, W. (2020). Aprendizaje Servicio en escuelas argentinas de Bachillerato Internacional. Alteridad. Revista de Educación, 16(1), 65-75. doi:https://doi.org/10.17163/alt.v16n1.2021.05

Ministerio de Educación. (2010). Actualización y fortalecimiento curricular de la Educación Genral Básica 2010. Quito, Ecuador: Ministerio de Educación. Recuperado el 16 de mayo de 2021, de https://educacion.gob.ec/wpcontent/uploads/downloads/2012/08/AC_2.pdf

Ministerio de Educación. (05 de julio de 2011). Acuerdo No. 242-11. Normativa para la implementación del nuevo currículo del Bachiullerato. Obtenido de https://educacion.gob.ec/wp-content/uploads/downloads/2014/02/ACUERDO242-11.pdf

Ministerio de Educación. (2016a). El perfil del bachiller ecuatoriano: desde la educación hacia la sociedad. Quito, Pichincha, Ecuador: Ministerio de Educación. Recuperado el 16 de mayo de 2021, de https://educacion.gob.ec/wpcontent/uploads/downloads/2016/12/perfil-del-bachiller.pdf

Ministerio de Educación. (17 de febrero de 2016b). Acuerdo Nro. MINEDUC-ME-201600020-A. Obtenido de https://educacion.gob.ec/wpcontent/uploads/downloads/2016/02/MINEDUC-ME-2016-00020-A.pdf 
Ministerio de Educación. (2019). Currículo de los Niveles der Educación Obligatoria. Nivel Bachillerato (Segunda ed., Vol. 2). Quito, Ecuador: Ministerio de Educación. Recuperado el 3 de junio de 2021, de Ministerio de Educación: https://educacion.gob.ec/wp-content/uploads/downloads/2019/09/BGU-tomo2.pdf

Ministerio de Educación. (2021). Instituciones 2019-2020. Obtenido de https://educacion.gob.ec/visualizador-estadistico/

Niño Zafra, L. S. (2013). El currículo y la evaluación críticos: ¿Del control y la rendición de cuentas a la autonomía y la democracia? En L. S. Niño Zafra (Ed.), Currículo y evaluación críticos: pedagogía para la autonomía y la democracia (Primera ed., págs. 11-30). Bogotá, Colombia: Universidad Pedagógica Nacional. doi:https://doi.org/10.2307/j.ctvfc52sz

Ortiz Ocaña, A. (2014). Currículo y didáctica. Bogotá, Colombia: Ediciones de la U. Recuperado el 17 de mayo de 2021

Perlo Pachega, C. L. (2019). La pedagogía biocéntrica: del currículum disciplinarionormativo al reencantamiento de la educación. Revista Scientific, 4(12), 68-88. doi:https://doi.org/10.29394/Scientific.issn.2542-2987.2019.4.12.3.68-88

Sandoval Rubilar, P. R., Frit Carrillo, M., Maldonado Fuentes, A. C., \& Rodríguez Alveal, F. (2010). Evaluación de habilidades en matemática y comprensión lectora en estudiantes que ingresan a pedagogía en educación básica: un estudio comparativo en dos universidades del Consejo de Rectores. Educar em Revista(2), 73-102. Recuperado el 27 de mayo de 2021, de https://www.redalyc.org/pdf/1550/155021686005.pdf

Tinto Arandes, J. A. (2013). El análisis de contenido como herramienta de utilidad para la realización de una investigación descriptiva. Un ejemplo de aplicación práctica utilizado para conocer las investigaciones realizadas sobre la imagen de marca de España. Provincia(29), 135-173. Recuperado el 2 de junio de 2021, de https://www.redalyc.org/pdf/555/55530465007.pdf

Vega Vega, J. C., Niño Duarte, F., \& Cárdena, Y. P. (2015). Enseñanza de las matemáticas básicas en un entorno e-Learning: un estudio de caso de la Universidad Manuela Beltrán Virtual. Revista Escuela de Administración de Negocios(79), 172-185. Recuperado el 26 de mayo de 2021, de http://www.redalyc.org/articulo.oa?id=20643042011

Vílchez Quesada, E., \& Ávila Herrera, J. F. (2021). Enseñanza y aprendizaje de la matemática para informática empleando documentos con un formato computable (CDFs): una percepción docente en la Universidad Nacional de Costa Rica. Revista digital Matemática, Educación e Internet, 21(2), 1-10. Recuperado el 26 de mayo de 2021, de https://revistas.tec.ac.cr/index.php/matematica/article/view/5608/5386 


\section{PARA CITAR EL ARTÍCULO INDEXADO.}

Mantilla-Falcón, L. M., Romero-Castro, D. I., Fonseca-Gómez, E. A., \& Mantilla-Falcón, M. S. (2021). Matemáticas en el bachillerato internacional y bachillerato general unificado de Ecuador. Un estudio crítico. Ciencia Digital, 5(4), 46-71. https://doi.org/10.33262/cienciadigital.v5i4.1867

\section{Liencia}

El artículo que se publica es de exclusiva responsabilidad de los autores y no necesariamente reflejan el pensamiento de la Revista Ciencia Digital.

El artículo queda en propiedad de la revista y, por tanto, su publicación parcial y/o total en otro medio tiene que ser autorizado por el director de la Revista Ciencia Digital.
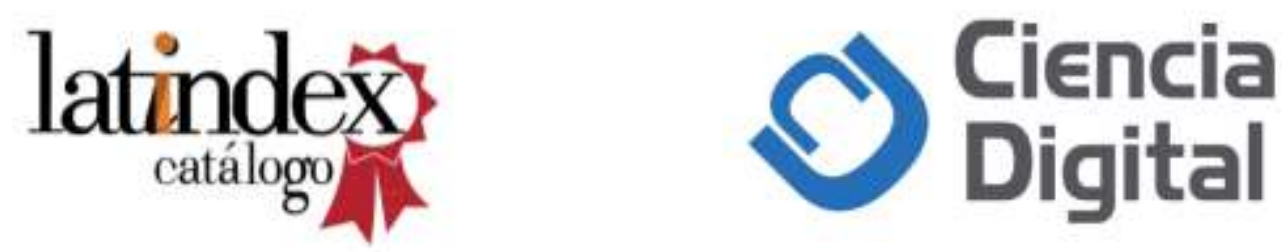